Radioterapia guiada por imagen. Impacto clínico

\title{
Clinical impact of image guided radiotherapy
}

\author{
P. Romero, E. Villafranca, M. Rico, A. Manterola, M.T. Vila, M. A. Domínguez
}

\section{RESUMEN}

La radioterapia guiada por imagen (RTGI) es un concepto que engloba la manera más moderna de administrar el tratamiento radioterápico El objetivo es maximizar la dosis depositada en el volumen a tratar (target), minimizando la dosis en los órganos sanos.

Esto no sería posible sin el continuo desarrollo tecnológico y de los software, sobre todo en las siguientes áreas: registrar imágenes deformables, replanificar nuevos tratamientos, imagen en tiempo real y cálculo de dosis acumulada.

El impacto clínico es evidente, pero poco se habla del impacto en la reorganización de los servicios de Oncología Radioterápica. La RTGI supone un entrenamiento de todo el equipo involucrado, con un periodo de aprendizaje y puesta en marcha. Con la experiencia adquirida, el tiempo dedicado a cada paciente (en todas las etapas de su tratamiento: simulación, planificación, puesta en marcha, sistemas de verificación de posicionamiento, correcciones on-line, off-line, replanificación, controles clínicos periódicos), es muy superior al que se precisa en la radioterapia convencional, motivo por el que aparecen nuevas responsabilidades y roles.

Palabras clave. Radioterapia guiada por imagen. Radioterapia adaptativa. Cáncer de próstata. Cone beam CT. Fiduciales.

\begin{abstract}
Image guided radiotherapy (IGR) is a concept that encompasses the most modern way of administering radiotherapy treatment. The aim is to maximise the dose deposited in the target volume, minimising the dose in healthy organs.

This would not be possible without the continuous development of technology and software, above all in the following areas: deformable image registration, replanning new treatments, real time image and calculation of accumulated dose.

While the clinical impact is evident, little is said about the impact on the reorganisation of the Radiotherapy Oncology services. IGR supposes training all team members involved, with a training and a starting period. With the experience acquired, the time dedicated to each patient (in all stages of treatment: simulation, planning, starting out, systems for verifying position, on-line, off-line corrections, replanning, periodic clinical controls) is far higher than that required in conventional radiotherapy, which gives rise to new responsibilities and roles.
\end{abstract}

Key words. Image guided radiotherapy. Adaptive radiotherapy. Prostate cancer. Cone beam CT. Fiducials.
Servicio de Oncología Radioterápica. Hospital de Navarra. Pamplona.

\section{Correspondencia \\ Pilar Romero Rojano}

Servicio de Oncología Radioterápica

Hospital de Navarra

Irunlarrea, 3

31008 Pamplona

Tfno: 848428475

E-mail: mp.romero.rojano@cfnavarra.es 


\section{INTRODUCCIÓN}

La radioterapia es una de las principales modalidades terapéuticas que existe contra el cáncer. De una manera muy sencilla, el proceso de planificación del tratamiento radioterápico consiste en delimitar el volumen tumoral (target), denominado GTV (gross treatment volumen), CTV (clinical target volumen) y los órganos a riesgo (oar) (ICRU 62).

En el proceso de planificación y administración del tratamiento radioterápico, existen unas incertidumbres sistemáticas y aleatorias. Las primeras son debidas a errores en la planificación y simulación (diferente posicionamiento del paciente durante la adquisición de las imágenes para planificar el tratamiento y durante el posicionamiento del tratamiento). Estos errores sistemáticos se repetirán durante todo el tratamiento. Los errores aleatorios aparecen por las desviaciones diarias al colocar al paciente, por cambios anatómicos en la morfología del tumor o por el movimiento interno que presentan algunos órganos (Ej. el pulmón con los movimientos respiratorios). Los errores aleatorios se producen en el momento y durante la administración del tratamiento.

Estas incertidumbres son las responsables de la existencia del $\mathrm{PTV}^{1}(P T V$ : planning target volumen), que es el margen de seguridad adecuado que hay que proporcionar al target para garantizar que recibe la dosis prescrita.

En la radioterapia convencional, la verificación de posicionamiento se realiza con imágenes planas de megavoltaje basándose en referencias óseas, sin visualizar los tejidos blandos (target y/o oar), aumentando así las incertidumbres. Por eso los PTV entran en conflicto con los oar, limitando así la escalada de dosis.

La RTGI supone un avance en precisión y mejora de la habilidad del oncólogo radioterápico para aumentar la dosis de una manera segura y eficaz. Ayuda a localizar y enfocar el target con una velocidad y precisión adecuadas, permitiendo modificar con exactitud y adaptar el tratamiento diariariamente $\mathrm{e}^{2,3}$.

\section{SISTEMAS DE IMAGEN GUIADA}

Debido a los cambios anatómicos que experimenta el target, es necesario obtener imágenes que aseguren la precisión del tratamiento. Estas imágenes pueden ser:

- Imágenes planas en 2D: electronic portal imaging devices de megavoltaje o kilovoltaje (Kv) para visualizar marcadores radioopacos.

- Imágenes volumétricas: ecografía, tomografía computarizada.

- Imágenes 4D (obtener información anatómica en referencia a los cambios producidos por la respiración) en tiempo real, en la sala de tratamiento, es el fundamento de la RTGI.

Cuando se obtiene una imagen hay que compararla con la de referencia (suele ser una imagen plana radiográfica de $\mathrm{Kv}$, que equivale a la reconstrucción digital radiográfica (RDR), generada en el planificador a partir de la TC de simulación. Esta imagen de referencia o las imágenes de la TC de simulación son las que se utilizan para comparar y verificar el posicionamiento del paciente o del target en el momento de administrar el tratamiento radioterápico. Si se compara justo en el momento de realizarla y previamente a iniciar el tratamiento, se denomina verificación on line. Las modificaciones realizadas en este momento, corrigen los errores sistemáticos y aleatorios, por eso, el día del inicio y en los cambios de fase, siempre se realiza verificación on line. Los protocolos de corrección on line, exigen la presencia del oncólogo radioterápico, quien decide la modificación, y determina el momento y número de verificaciones on line. Si la verificación de la imagen se realiza una vez administrado el tratamiento, se denomina verificación off line. Este tipo de verificaciones corrige los errores sistemáticos estimados durante la fase inicial del tratamiento en las fases posteriores. Los protocolos off line son los más utilizados, los denominados shinrinking action level (SAL) y no action level (NAL).

La comparación de estas imágenes es fundamental y puede llevar a no tener que realizar ningún desplazamiento; el otro extremo es tener que replanificar el trata- 
miento por cambios importantes en la geometría del target $^{5}$, o movimiento diario del mismo. La posibilidad de medir y gestionar estos errores, dependerá del sistema de RTGI empleado. A continuación se describe los principales sistemas de imagen guiada:

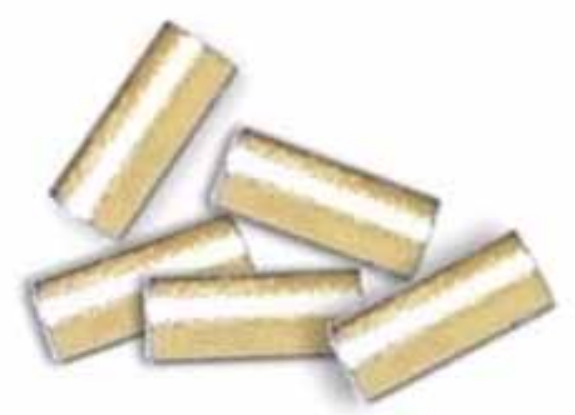

Figura 1. Ejemplo de marcadores fiduciales de oro de $5 \mathrm{~mm}$ de longitud y $2 \mathrm{~mm}$ diámetro.

1. Marcadores radioopacos (fiduciales) de diferentes materiales (oro, plata), dimensiones (2 mm-10 $\mathrm{mm}$ ) y formas (esferas, semillas cilíndricas) (Fig. 1), implantados en tejido blando permiten visualizar directamente el target. La principal aplicación clínica es el tratamiento del tumor de próstata ${ }^{4}$, también en páncreas y pulmón. Se identifican fácilmente en imagen plana radiográfica, facilitando la decisión de corregir el posicionamiento on line y la rapidez en la obtención de la imagen. El principal problema es que pueden migrar y la ausencia de información con respecto a posibles deformaciones de los tejidos que sostienen al tumor u oar cercanos.

2. Ecografía. Se obtienen imágenes volumétricas. Su principal aplicación clínica es en el tumor de próstata. Presenta como principal ventaja su inocuidad y la fácil visualización de la próstata. Los inconvenientes son: proporciona imágenes diferentes a las de la TC del simulador, variabilidad importante interobservador y la posible modificación de la morfología de la próstata con la presión de la próstata.

3. Cone beam tomografía computarizada (CBCT) (Fig. 2). Permite obtener imágenes de tomografía axial en la sala de tratamiento, facilitando la comparación con las imágenes de la TC de simulación. Consiste en un equipo de TC de haz cónico (cone beam) montado en el brazo del acelerador lineal, que obtiene las imágenes volumétricas en una sola rotación del tubo de rayos $\mathrm{X}$ y del detector. Su principal problema es el largo tiempo de adquisición de las imágenes (aproximadamente un minuto) y que la respiración pulmonar artefacta mucho las imágenes sobre todo las de tórax y abdomen.

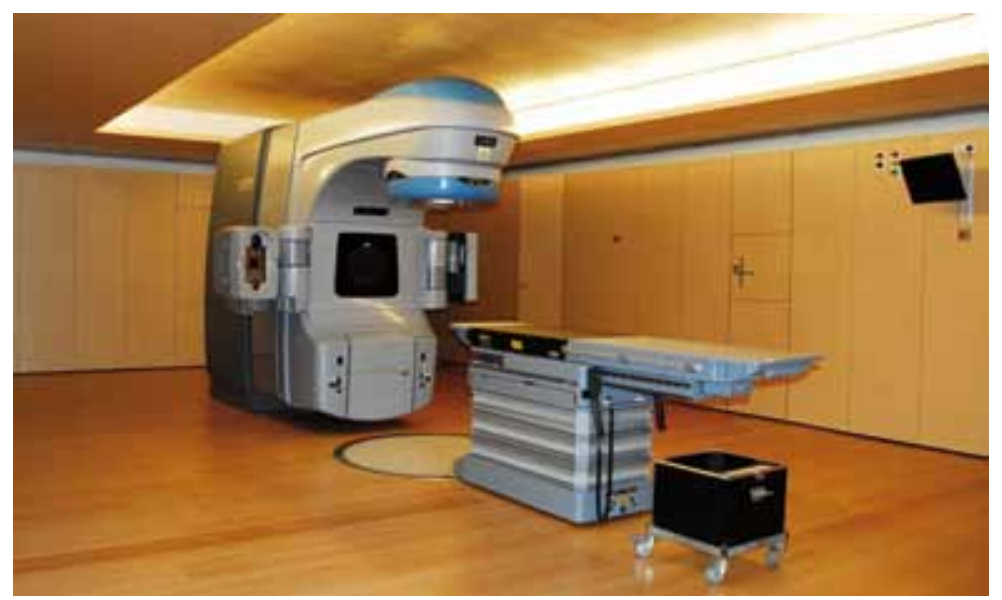

Figura 2. Acelerador lineal con dispositivo de Cone beam CT de kilo y megavoltaje. 
4. En estudio: sistemas guiados por fluoroscopia, tomosíntesis y sistemas electromagnéticos. Muy interesante el futuro de la integración de imágenes de resonancia magnética en el tratamiento radioterápico ${ }^{5}$.

\section{APLICACIONES CLÍNICAS}

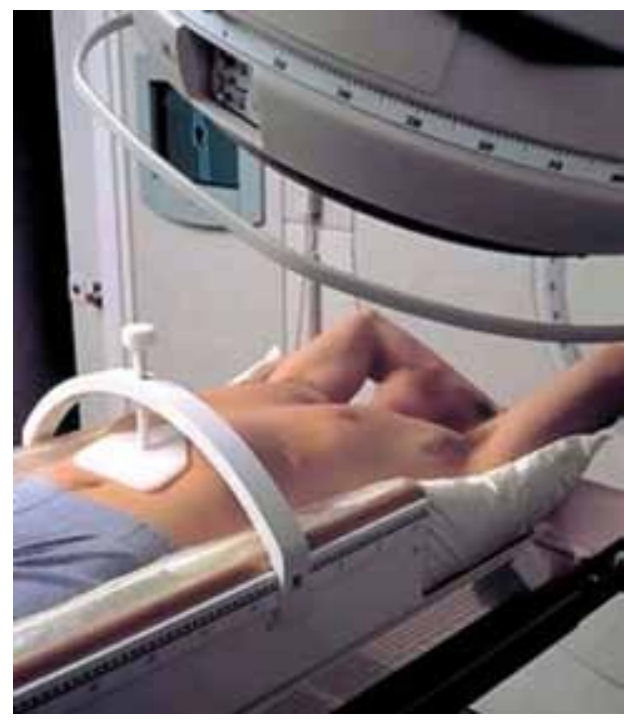

Figura 3. Ejemplo de inmovilizador body frame.

Comparando el error residual (la desviación que permanece después de corregir el posicionamiento desde la posición planificada) con cada sistema de imagen guiada en los tumores de próstata ${ }^{6}$, el que menos error residual genera (de unos $2 \mathrm{~mm}$ ) es la imagen diaria con CBCT con referencia de tejido blando y CBCT con fiduciales. En el caso de tumor de pulmón ${ }^{7}$, los movimientos respiratorios complican la obtención de las imágenes. El error residual, con un esquema hipofraccionado e inmovilización body frame, es un inmovilizador que aporta un sistema de presión abdominal para disminuir el desplazamiento debido al movimiento respiratorio (Fig. 3), e imágenes diarias de CBCT con referencia del tumor. Se obtienen errores de $3 \mathrm{~mm}$ (promedio de la posición del tumor con respecto a la respiración). En los tumores de cabeza y cuello $^{8}$, el error residual, usando imágenes radiográficas, es de $>5 \mathrm{~mm}$ en el $10 \%$ de los pacientes si se realiza diariamente, en cambio, si se realiza semanalmente, este error se objetiva en el $33 \%$ de los pacientes. En este tipo de tumores, es importante la replanificación ${ }^{9}$, debido a que existe una disminución del tamaño del tumor (media del $69 \%$ ), del tamaño de las glándulas parótidas (28\%) y de los ganglios, desplazando el posicionamiento de órganos sanos a zonas de altas dosis. Esto adquiere más importancia si cabe, cuando se utiliza técnica de radioterapia de intensidad modulada.

Al combinar la RTGI y la radioterapia de intensidad modulada se tienen las herramientas necesarias para administrar altas dosis/fracción, el llamado hipofraccionamiento, acortando así el tiempo del tratamiento y garantizando un tratamiento eficaz y seguro. Los protocolos de verificación on line son clave en este tipo de tratamientos. Existen estudios clínicos ${ }^{10}$ en marcha pendientes de resultados definitivos. Las aplicaciones clínicas más estudiadas son en las lesiones paraespinales ${ }^{11}$, tumor de pulmón ${ }^{12} \mathrm{y}$ tumor intrahepático ${ }^{13}$. En las lesiones paraespinales, se compara el error residual entre las imágenes planas de $\mathrm{kv}$ con las del CBCT, siendo de $2,3 \mathrm{~mm}$ y 1,5 $\mathrm{mm}$ respectivamente. Con el $\mathrm{CBCT}$, se detectan mejor las rotaciones, aún con los inmovilizadores en "cuna" de los pacientes. En el caso de las lesiones intrahepáticas, también se observa una ventaja en el error residual de la imagen volumétrica con el CBCT con respecto a la imagen plana, mejorando la dosis en las estructuras vasculares y conductos intrahepáticos sanos. El control de calidad de estos equipos debe ser exhaustivo, incluyendo tolerancias y periodicidad $^{14}$.

\section{CONSIDERACIONES GENERALES}

La exactitud del posicionamiento del target está influenciado por el sistema de imagen guiada y el protocolo de corrección. Con la RTGI, se dan unas condiciones para planificar los tratamientos con un alto índice de conformación y alcanzar con 
gran precisión la localización del target y delimitación de los volúmenes de interés.

Para realizar cambios en la práctica clínica, como disminuir el tamaño del PTV, aumentar la dosis total o instaurar tratamientos hipofraccionados, hay que identificar y controlar las incertidumbres y asegurar su reproducibilidad durante el tratamiento. La calidad de las imágenes que se obtienen con los diferentes sistemas de IGRT son clave para poder realizar correcciones de posicionamiento, y adaptar el tratamiento planifidado inicialmente a las modificaciones diarias.

Todo lo comentado no sería posible sin el equipo formado por: oncólogos radioterápicos, radiofísicos, dosimetristas, técnicos en radioterapia y enfermería.

La reorganización del servicio de Oncología Radioterápica, es estrictamente necesaria con la puesta en marcha de RTGI. Los tiempos necesarios para todo el proceso del tratamiento radioterápico (simulación, planificación, protocolos de corrección on line y off line, replanificación, control periódico de los pacientes), son superiores a los de la radioterapia convencional. Aparecen nuevos roles y nuevas responsabilidades que afectan a todo el personal involucrado en el proceso del tratamiento radioterápico, en su planificación, puesta en marcha y seguimiento de los pacientes.

Debido al importante impacto clínico que supone la RTGI, conjuntamente con la técnica de modulación de dosis, hay que confirmar el beneficio clínico, que debe ser demostrado en los diversos estudios randomizados que están en curso de presentar resultados.

\section{BIBLIOGRAFÍA}

1. Bortfeld T, Jiang SB, Rietzel E. Effects of motion on the total dose distribution. Semin Radiat Oncol 2004; 14: 41-51.

2. JAFFRAY DA. Image-guided radiation therapy: from concept to practice. Semin Radiat Oncol 2007; 17: 243-244.

3. Shirato H, Shimuzu S, Kunieda T, Kitamura K, Van Herk M, KAGEI K et al. Physical aspects of real time tumor tracking system for gated radiotherapy. Int J Radiat Oncol Biol Phys 2000; 48: 1187-1195.
4. van der Heide A, Kotte AN, Denhad H, Hofman P, LAGENIJK JJ, VAN VuLPEN M. Analysis of fiducial marker-based position with prostate verification in the external beam radiotherapy of prostate cancer. Radiother Oncol 2007; 82: 38-45.

5. RaAymakers BW, RaAjmaKers AJ, Kotte AN, LAGENDIJK JJ. Integrating a MRI scanner with a 6 MV radiotherapy accelerator. Dose deposition in a transverse field. Phys Med Biol 2004; 49: 4109-4118.

6. Serago CF, Buskirk SJ, Igel TC, Gale AA, Serago NE, EARLE JD. Comparison of daily megavoltaje electronic portal imaging or kilovoltaje imaging with marker seeds to ultrasound imaging or skin marks for prostate localization and treatment positioning in patients with prostate cancer. Int J Radiat Oncol Biol Phys 2006; 65: 1585-1592.

7. Chang J, Mageras G, Yorke E, De Arruda F, SiLLANPAA J, RosENZWEIG KE et al. Observation of interfractional variations in lung tumor position using respiratory gated and ungated magavoltaje cone-beam computed tomography. Int $\mathrm{J}$ Radiat Oncol Biol Phys 2007; 67: 1548-1558.

8. Zeidan OA, Langen KM, Meeks SL, Manon RR, WAGNER TH, WilloughBY TR et al. Evaluation of image-guiadance protocols in the treatment of head and neck cancers. Int J Radiat Oncol Biol Phys 2007; 67: 670-677.

9. Kuo C, Wu TH, Chung TS, Huang KW, Chao KS, $\mathrm{Su}$ WC et al. Effect of regression of enlargedneck lymph nodes on radiation doses received by parotid glands during intensitymodulated radiotherapy for head and neck cancer. Am J Clin Oncol 2006; 29: 600-605.

10. Carey Sampson M, Katz A, Constine LS. Stereotactic body radiation therapy for extracranial oligometastasis: Does the sword have double edge? Semin Radiat Oncol 2006; 16 : 67-76.

11. Yamada Y, Lovelock M, BiLSKY MH. Image-guided intensity-modulated radiation therapy of spine tumors. Curr Neurol Neurosci Rep 2006; 6: 207-211.

12. Timmerman RD, Forster KM, Chinsoo Cho L. Extracranial stereotactic radiation delivery. Semin Radiat Oncol 2005; 15: 202-207.

13. Balter M, Brock KK, Litzenberg DW, Mc shan DL, LAWREnce TS, TEn HAKEn R et al. Daily targeting of intrahepatic tumors of radiotherapy. Int $\mathrm{J}$ Radiat Oncol Biol Phys 2002; 52: 266-271.

14. Bissonnentette JP. Quality assurance of imageguidance technologies. Semon Radiat Oncol 2007; 17; 278-286. 
